\title{
Contents of the Standardized Suicide Prevention Program for Gatekeeper Intervention in Korea, Version 2.0
}

\author{
Kyoung-Sae Na ${ }^{1 *}$, Seon-Cheol Park ${ }^{2 \star}$, Sun-Jung Kwon ${ }^{3}$, Minjae Kim ${ }^{4}$, Hyoung-Jun Kim ${ }^{5}$, Myungjae Baik ${ }^{6}$, \\ Jinmi Seol ${ }^{5}$, Eun Ji An ${ }^{5}$, Sang Min Lee ${ }^{7}$, Eun-Jin Lee ${ }^{8}$, Meerae Lim ${ }^{5}$, Sung Joon $\mathrm{Cho}^{9}$, Gwang Hun $\mathrm{Kim}^{10}$, \\ Nari Kim ${ }^{7}$, Hong Jin Jeon ${ }^{11}$, Jong-Woo Paik ${ }^{7,12}$, Kang Seob Oh ${ }^{\text {, }}$ and Hwa-Young Lee ${ }^{13 凶}$ \\ 1Department of Psychiatry, Gachon University College of Medicine, Gil Medical Center, Incheon, Republic of Korea \\ ${ }^{2}$ Department of Psychiatry, Inje University Haeundae Paik Hospital, Busan, Republic of Korea \\ ${ }^{3}$ Suicide Prevention Center, Republic of Korea Air Force, Daejeon, Republic of Korea \\ ${ }^{4}$ Department of Neuropsychiatry, Seoul National University Hospital, Seoul, Republic of Korea \\ ${ }^{5}$ Workplace Mental Health Institute, Kangbuk Samsung Hospital, Sungkyunkwan University School of Medicine, Seoul, Republic of Korea \\ ${ }^{6}$ Gyeonggi Provincial Mental Health Center, Suwon, Republic of Korea \\ ${ }^{7}$ Department of Psychiatry, Kyung Hee University College of Medicine, Seoul, Republic of Korea \\ ${ }^{8}$ Department of Social Welfare, Suwon Science College, Hwaseong, Republic of Korea \\ ${ }^{9}$ Department of Psychiatry, Kangbuk Samsung Hospital, Sungkyunkwan University School of Medicine, Seoul, Republic of Korea \\ ${ }^{10} \mathrm{Korea}$ Association for Suicide Prevention, Seoul, Republic of Korea \\ ${ }^{11}$ Department of Psychiatry, Samsung Medical Center, Sungkyunkwan University School of Medicine, Seoul, Republic of Korea \\ ${ }^{12}$ Korea Suicide Prevention Center, Seoul, Republic of Korea \\ ${ }^{13}$ Department of Psychiatry, Soonchunhyang University Cheonan Hospital, Cheonan, Republic of Korea
}

Objective Suicide is a huge nationwide problem that incurs a lot of socio-economic costs. Suicide also inflicts severe distress on the people left behind. The government of the Republic of Korea has been making many policy efforts to reduce suicide rate. The gatekeeper program, 'Suicide CARE', is one of the meaningful modalities for preventing suicide.

Methods Multidisciplinary research team collaborated to update the 'Suicide CARE' to version 2.0.

Results In the 'Introductory part', the authors have the time to think about the necessity and significance of the program before conducting full-scale gatekeeper training. In the 'Careful observation' part, trainees learn how to understand and recognize the various linguistic, behavioral, and situational signals that a person shows before committing suicide. In the 'Active listening' part, trainees learn how to ask suicide with a value-neutral attitude as well listening empathetically. In the 'Risk evaluation and Expert referral' part, trainees learn intervening strategies to identify a person's suicidal intention, plan, and past suicide attempts, and connect the person to appropriate institutes or services.

Conclusion Subsequent studies should be conducted to verify the efficacy of the gatekeeper program.

Psychiatry Investig 2020;17(11):1149-1157

Key Words Suicide, Prevention, Gatekeeper, Mental health, Public health.

\section{INTRODUCTION}

Suicide is to lead to death by deliberately harming oneself with intent to die. Suicide does not end with taking one life.
It is usually known that when one person commits suicide, there are more than six close family and friends left behind. ${ }^{1}$ Suicide causes significant psychiatric symptoms such as depression, anxiety, and complicated grief to those left behind. ${ }^{2,3}$

Received: July 13, 2020 Revised: October 30, 2020 Accepted: November 2, 2020

$\triangle$ Correspondence: Jong-Woo Paik, MD, PhD

Department of Psychiatry, Kyung Hee University College of Medicine, 23 Kyungheedae-ro, Dongdaemun-gu, Seoul 02453, Republic of Korea

Tel: +82-2-958-8419, Fax: +82-2-957-1997, E-mail: paikjw@khu.ac.kr

$\triangle$ Correspondence: Hwa-Young Lee, MD, PhD

Department of Psychiatry, Soonchunhyang University Cheonan Hospital, 31 Suncheonhyang 6-gil, Dongnam-gu, Cheonan 31151, Republic of Korea

Tel: +82-41-570-3871, Fax: +82-41-592-3804, E-mail: leehway@gmail.com

*These authors contributed equally to this work.

(c) This is an Open Access article distributed under the terms of the Creative Commons Attribution Non-Commercial License (https://creativecommons.org/licenses/by-nc/4.0) which permits unrestricted non-commercial use, distribution, and reproduction in any medium, provided the original work is properly cited 
Suicide also has a serious social and economic impact. In an Australian workplace suicide study, the economic cost of suicide and non-fatal suicidal behavior was reported to reach $\$ 6.73$ billion. ${ }^{4}$ In the United States, the total national cost of suicide attempts was $\$ 58.4$ billion and if the under-reported ones are corrected, the cost was reported to total $\$ 93.5$ billion or $\$ 298$ per population. ${ }^{5}$

Although the suicide rates tend to decrease worldwide since the $1990 \mathrm{~s}^{6}{ }^{6}$ and there are claims that the proportion of suicide that contributes to mortality is less than previously known, ${ }^{7}$ suicide remains a serious national problem that needs to be resolved urgently in some countries and cultures. One of the representative countries is the Republic of Korea. The suicide rate of the Republic of Korea in 2018 was 26.6 per 100,000 population, an increase of 2.3 (9.5\%) compared to $2017 .{ }^{8}$ This is also the highest level among the countries in the Organization for Economic Cooperation and Development. ${ }^{9}$

The Republic of Korea has implemented many strategic policies to lower suicide rates. On March 30, 2011, 'Act on the Prevention of Suicide and the Creation of Culture of Respect for Life' was enacted. ${ }^{10}$ The purpose of this Act is to protect the precious lives of the people and foster a culture of respect for life by defining necessary matters regarding national responsibility and prevention policies for suicide. According to this Act, the Korea Suicide Prevention Center was opened in 2011 and the Korea Psychological Autopsy Center was also opened in 2014. The Korean government announced the 'National Action Plan for Suicide Prevention (2018-2022)' after a joint meeting of related ministries on January $23,2018 .{ }^{11}$ According to the national action plan, Suicide Prevention Policy Department, a department dedicated to suicide prevention, was separately established within the Bureau of Health Policy in the Ministry of Health \& Welfare in February 2018. In addition, the national budget for suicide prevention has increased from 8.5 billion won in 2016 to 9.9 billion won in 2017 and 16.8 billion won in 2018. However, Korea has meager budget for the prevention of suicide. The per capita budget for the prevention of suicide in Korea is still 326 won, which is only onetwentieth that of Japan, which is 6,200 won. ${ }^{11}$

Whereas it is needed to increase the amount of budget, it is also essential to use a budget efficiently. The gatekeeper program occupies a significant part of the national suicide prevention policy. The gatekeeper program aims to enhance the ability of gatekeepers to determine the level of risk of suicide by acquiring knowledge, attitudes, and skills to identify those who have the risk of suicide, and to connect them to appropriate services. ${ }^{12}$ Indeed, the gatekeeper program is included in the ten tasks of 'the National Suicide Prevention Action Plan (2018-2022), and making a gatekeeper training system for the entire nation is being implemented as one of 20 detailed tasks. ${ }^{11}$
The 'Careful observation, Active listening, Risk evaluation and Expert referral (Suicide CARE)', which is the standardized suicide prevention program for gatekeeper intervention in Korea, was first developed in Korea in December 2012. ${ }^{13}$ The first version was 1.0. In 2014, 'Suicide CARE' was updated to version 1.6. ${ }^{14}$ After that, 'Suicide CARE' began to apply life cycle approach. The 'Suicide CARE: version 1.6A' was developed for teenagers in 2015, ${ }^{15}$ and 'Suicide CARE: version 1.6W' was developed for office workers in 2016. ${ }^{16}$ According to the goal in the national suicide prevention action plan, a total of 1 million gatekeepers have been trained by the national gatekeeper programs in Korea by $2018 .{ }^{17}$

Since 'Suicide CARE' was first developed in 2012, many evidence-based results, which could be the basis for establishing and implementing strategies for suicide prevention, have been reported over the past seven years. In addition, after the Korea Psychological Autopsy Center opened in 2014, various signals that the suicide victims had shown while they were alive began to be identified, so it became possible to analyze them scientifically. On the other hand, as 'Suicide CARE' version 1.6 has been widely distributed to local communities in various regions and conditions, a lot of feedback from the field has been collected. Based on all the various grounds and opinions collected in this way, there have been growing calls for updating the existing version of 'Suicide CARE' version 1. Therefore, the development of 'Suicide CARE' version 2.0 was begun in August 2019, and the development was completed in December 2019. ${ }^{1}$

There are many gatekeeper programs around the world. ${ }^{18}$ However, there would be no such program as that of Korea's 'Suicide CARE' in which the government creates a standardized gatekeeper program, continuously updates it, actively distributes and utilizes it, and trains about $2 \%$ of the entire citizens as gatekeepers. 'Suicide CARE' is the fruit of the Korean government's steady efforts to lower suicide rates through the systematic implementation of such suicide prevention policies. Therefore, it is meaningful for Korea to share the contents of 'Suicide CARE' with other countries in the world. However, whereas 'Suicide CARE' has been regarded as one of the representative suicide prevention programs in Korea, it has been unknown to foreign countries or has been introduced incorrectly. Suicide CARE is called 'Bogo Deudgo Malhagi' in Korean, abbreviated as 'Bo-Deud-Mal'. Even though this is a Korean standard suicide prevention program developed and distributed by the government, the English translation has not been consistent. Some researchers translated 'Bogo Deudgo Malhagi' into 'Watch, Listen and Talk.'19 In the 'Mental Health ATLAS 2017' published by the World Health Organization, it was translated into 'Seeing, Listening, and Speaking. ${ }^{20}$ 


\section{METHODS}

Given the above consideration, multidisciplinary research team updated the Suicide CARE to version 2.0 and wrote an introductory paper to spread the official English name and essential components to multidisciplinary researchers related to suicide prevention worldwide. The detailed description for the development process of the Suicide CARE has been reported and published separately in a recent article. ${ }^{21}$ The authors introduced 'Suicide CARE' version 2.0 according to the composition of 'Introductory part', 'Careful observation', 'Active listening', and 'Risk evaluation and Expert referral'. The author also looked at what is needed and what needs to be improved for the most useful use of suicide prevention policy tools such as 'Suicide CARE' version 2.0.

\section{RESULTS}

\section{The 'introductory part'}

The purpose of the introductory part is to think about its necessity and significance before we go into full-scale gatekeeper training. This part is mainly composed of Lecture Overview, Introductory Video, Comparison between Suicide and Traffic Accidents, Unknown Suicides, and Necessity of Gatekeeper Program. In the "Introductory Video", the current impact of suicide in Korea is introduced, and it is taught that many suicides are preventable. In "Comparison between Suicide and Traffic Accidents," the changes in suicide deaths and traffic accident deaths over the past few decades were compared to each other. Traffic accident deaths have fallen to about one-third for about 20 years thanks to a steady investment by the Korean government, while suicide deaths have nearly doubled for the same period. The authors suggested that public interest, social awareness, systematic planning, and sufficient investment can lead to good results through comparisons of traffic accident deaths and suicide deaths. "Unknown Suicides" refers to suicides that are not reflected in statistical figures. In this section, it is suggested that there are $5 \%$ of the entire population with the cases in which even if it is not counted as official suicide, it can be said to be actual suicide. It is also emphasized that suicide is never a rare or of little important event in our society. Lastly, the "Necessity of Gatekeeper Program" includes that the World Health Organization and the US Centers for Disease Control and Prevention recognize the gatekeeper program as an effective intervention strategy for suicide prevention.

\section{The 'careful observation'}

In the 'Careful observation' part, we deal with detecting signals from high-risk people at suicide. This part consists of eight parts (Table 1). The contents of this part were updated largely through the active use of the results of the '2018 Psychological Autopsy Interview' by the Central Psychological Autopsy Center. The resources provided by the Central Psychological Autopsy Center made it possible to reflect as much of the danger signals that real suicide victims have shown in their lives. The first section, “Three Examples," show situational and behavioral signals of suicides that those around people at risk for suicides may have perceived while they were alive. This section can remind us of the importance of timely perception of danger signals that suggest suicide. The "Undetected Suicide Signal" shows how suicide risk signals were not correctly detected in the real world by suggesting that the suicide victims expressed $92 \%$ suicide risk signals, but $77 \%$ of people around the suicide victims did not notice the signals. The "The Process of Reaching Suicide" section shows three stages of suicide, which are suicide thought, a suicide plan, and suicide attempt. The "Linguistic Signals" visually show the danger signals that suicide victims showed most in their lives: expressions of death including suicide, physical discomfort, and selfdeprecating words. In addition, suicide risk signals such as posting death-related content on social media, interest in the afterlife, and expressions of suicide are frequently presented. The "Behavioral Signals" show various behaviors of suicidal deaths including changes in emotions and behaviors before death, depressive symptoms, settling affairs before death, and death-related behaviors. In particular, the situation in which urgent intervention is needed was emphasized by highlighting the dangerous behaviors of suicides when suicides are imminent, such as three months or one week before suicide. The "Situational Signals" shows the situational difficulties suicide victims experienced during their lifetime, including mental health, work, economics, and family-related problems. In particular, the age group is divided into the young, the middleaged, and the elderly, respectively, and high-risk situational signals for the corresponding age group are presented. For example, academic and interpersonal issues such as study and date in the young, economic problems such as debt in the middle-aged, and chronic illness \& physical symptoms in the elderly are presented as dangerous situations. In the "Video," the examples of young people are adapted and the scenario is composed. In the last section, "What Should We Do After Careful Observation?", we take time to think about how the surrounding people should have responded after watching the video with the bad ending. Throughout this section, trainees are motivated to learn the next step, 'Active listening.'

\section{The 'active listening'}

Listening consists of nine parts (Table 2). First of all, the first "Video" shows linguistic, behavioral, and situational sig- 
Table 1. Components \& main contents of 'careful observation'

\begin{tabular}{|c|c|}
\hline Item & Core content \\
\hline Three examples & $\begin{array}{l}\text { - Three examples are provided in which people around a suicide have not noticed the various situational and } \\
\text { behavioral signals that the suicide victim sent during his lifetime. } \\
\text { - The 'Careful observation' section presents a topic by motivating trainees to recognize signals of suicide risk. }\end{array}$ \\
\hline $\begin{array}{l}\text { Undetected suicide } \\
\text { signal }\end{array}$ & $\begin{array}{l}\text { - We inform that } 92 \% \text { suicide deaths express suicide risk signals when they are alive, but } 77 \% \text { of those around them } \\
\text { are unaware of them. } \\
\text { - The importance to recognize suicide risk signals that people in high-risk groups repeatedly express is emphasized. }\end{array}$ \\
\hline $\begin{array}{l}\text { The process } \\
\text { of reaching suicide }\end{array}$ & $\begin{array}{l}\text { - Three suicide stages (suicide thought, suicide plan, and suicide attempt) are explained. } \\
\text { - Suicide thoughts only take up a very small part at first. If the person's beliefs, experiences, and precious things } \\
\text { are kept intact, they can prevent suicide thoughts from escalating into suicide plans or attempts to commit suicide. } \\
\text { - If the beliefs, experiences, and precious things that prevented suicide disappear, or if the suicide thought becomes } \\
\text { too large, it proceeds with a specific suicide plan. In this situation, the person can have a distorted conviction that his } \\
\text { only option is to commit suicide, as he cannot see his situation as a whole and his view becomes narrow. } \\
\text { - While the suicide plan is firm, the person has both hopelessness and impulsivity at the same time, eventually leading } \\
\text { to a suicide attempt. }\end{array}$ \\
\hline Linguistic signals & $\begin{array}{l}\text { - It is suggested that } 28 \% \text { of suicides frequently spoke about death such as suicide, } 26 \% \text { complained of physical } \\
\text { discomfort, and } 23 \% \text { said self-deprecating words. } \\
\text { - Suicides write down death-related expressions in letters, notes, SNS, etc., ask how to commit suicide, talk about } \\
\text { people who have committed suicide, and admire the afterlife. }\end{array}$ \\
\hline Behavioral signals & $\begin{array}{l}\text { - Suicides show behaviors such as unusual behavior, impulsive behavior, aggressive behavior, self-injury, and substance } \\
\text { abuse before attempting suicide. } \\
\text { - Other symptoms related to depression are also shown as follows. } \\
\text { - Changes in eating and sleeping } \\
\text { - Helplessness, social phobia, loss of interest in most daily life } \\
\text { - Difficulty in concentration and difficulty in making decisions about minor things } \\
\text { - Indifference to appearance management } \\
\text { - People often show the following actions before committing suicide. } \\
\text { - They settle their affairs and try to improve relationships. } \\
\text { - They give things that they have usually valued to other people. } \\
\text { They may also do the following actions directly related to death. } \\
\text { - They make a plan for suicide. } \\
\text { - They are overly immersed in poetry, music and movies related to death. } \\
\text { - This section provides a more timely identification of signals by presenting differences in behavioral signals according } \\
\text { to time, such as three months and one week before suicide. } \\
\text { - Danger signals three months before the suicide } \\
\text { - Changes in an emotional state } \\
\text { - Changes in eating habits } \\
\text { - Changes in sleep patterns } \\
\text { - Behaviors settling their affairs } \\
\text { - Helplessness, social phobia, loss of interest } \\
\text { - Danger signals one week before the suicide } \\
\text { - Unusual behavior, aggressive and impulsive behavior }\end{array}$ \\
\hline
\end{tabular}


Table 1. Components \& main contents of 'careful observation' (continued)

\begin{tabular}{|c|c|}
\hline Item & Core content \\
\hline tuational & $\begin{array}{l}\text { - Situational signals are closely related to life stress events. } \\
\text { - The average number of situational signals that suicide victims have shown in their lives is } 3.9 \text { on average. } \\
\text { - The main types of situational signals } \\
\text { - Mental health problems } \\
\text { - Work-related problems } \\
\text { - Economic problems } \\
\text { - Family-related problems } \\
\text { - Academic problems } \\
\text { - Health problems } \\
\text { - Couples and dating problems } \\
\text { - Interpersonal relationship problems }\end{array}$ \\
\hline Video 1 & $\begin{array}{l}\text { - Case of a young woman is presented. She has suicidal thoughts due to repeated employment failures and economic } \\
\text { difficulties } \\
\text { - Linguistic signals: verbally expressing exhausted and stressful situations, indirectly referencing to suicide, and saying } \\
\text { words to settle their human relationships. } \\
\text { - Behavioral signals: Looking hopeless, not doing well her part-time, difficulty in concentration, and changes in diet } \\
\text { and sleep, not able to keeping her appearance neat, avoiding interpersonal relationships, settling her relationships, } \\
\text { and posting pessimism on the social media. } \\
\text { - Eventually, people around her cannot be able to recognize these various signals. }\end{array}$ \\
\hline What should we do? & $\begin{array}{l}\text { - After checking videos that have failed to practice "Careful observation", trainees begin to think about what to do } \\
\text { afterwards. } \\
\text { - Motivating trainees to learn 'Active listening' }\end{array}$ \\
\hline
\end{tabular}

nals suggesting suicide from a self-employed middle-aged man. This video shows a surrounding person who fails to empathize with a person at risk of suicide. The surrounding person consequently fails to engage in suicide prevention intervention as a result of speaking and acting only according to his or her thoughts and judgments. This makes trainees think about how to respond initially to suicide high-risk group people. In the "Question 1 for listening: Do you think about suicide?" based on evidence-based research results, it emphasizes that trainees directly ask about suicide and asks a question about it because referring to suicide does not increase the danger of suicide, but rather helps to prevent it. The "The Listener's Mind" deals with various emotions that arise when it is directly confirmed that a person is thinking about suicide. The "Unhelpful Listening" shows that helpful interventions cannot be made if the listeners follow only their own emotions, not those of people at risk of suicide. In the "Question 2 for Listening: Why did you think about suicide?" We show that sympathizing with the minds of people at risk of suicide, not the listener's mind, is the most basic mindset. We show that sympathizing with people at risk of suicide begins by asking why they thought they should commit suicide. The "Listening Skill: Active Listening" shows how to create an atmosphere at which people at risk of suicide can comfortably tell his story through sympathetic listening and a non-judgmental attitude. The "The Response of Gatekeeper" is a section that trainees can practice the skills acquired in the "Listening" section. Intervention can be practiced naturally by the trainees actually doing the expressions such as "You were so hard that you wanted to die.", "It must have been a tough situation for you to tell the story, but thank you for telling me." The "Ambivalence" makes trainees know that someone who thinks of suicide also has a desire to live. The last section, "The Reason to Live," shows that people at risk of suicide can identify the reasons why they should live.

\section{The 'risk evaluation and expert referral'}

The 'Risk evaluation and Expert referral' consists of nine parts (Table 3). The first part, "Video 3," comprehensively shows the situational, behavioral, and linguistic signals of people at risk of suicide. The "Checking Suicide Risk" teaches questions accurately identifying suicide plans, methods, and prior suicide attempts. The "Helping safely" shows how to secure safety for people at risk of suicide. For example, it includes not recommending alcohol to people at risk of suicide. In the "Understanding Depression," we introduce mental illnesses such as depression and explain the symptoms. In the "Piece Together a Puzzle of Hope," we introduce people, institutions, and services that people at risk of suicide can call or use for help. In the "Core Contacts," we introduce Korea's representative suicide prevention services (e.g., 24-hour suicide prevention hotline 1577-0199). The "Good Ending" shows a finale that a selfemployed middle-aged man who was once at risk of suicide 
Table 2. Components \& main contents of the 'active listening'

\begin{tabular}{|c|c|}
\hline Item & Core content \\
\hline Video 2 & $\begin{array}{l}\text { - It is suggested that a middle-aged man who is self-employed wants to die due to financial difficulties. } \\
\text { - Linguistic signals: a direct reference to suicide } \\
\text { - Behavioral signals: paying attention to celebrity suicide news, excessive drinking, and frequent emotional } \\
\text { changes } \\
\text { - Situational signals: The shop sales are not good, he wanted to sell his shop, but it wasn't sold, and he has many } \\
\text { loan. } \\
\text { - Composing the story as below, which led to a failure of 'Active Listening' } \\
\text { - People approached him with worries and concerns, but they did not sympathize with him at risk of suicide, } \\
\text { and the people acted just as they felt. In other words, they were angry at him and presented a quick solution. }\end{array}$ \\
\hline $\begin{array}{l}\text { Question } 1 \text { for listening: } \\
\text { "do you think about } \\
\text { suicide?" }\end{array}$ & $\begin{array}{l}\text { - Trainees are educated to ask directly about suicide. } \\
\text { - By using the value-neutral word 'suicide', trainees can maintain a non-judgmental attitude and engage } \\
\text { in dialogue. } \\
\text { - By using the word suicide first, people around him create an atmosphere where the person in danger of suicide } \\
\text { can feel safe and talk. } \\
\text { - Since some of the trainees may be somewhat awkward asking directly about suicide, the practice in which they } \\
\text { can comfortably ask about suicide is supposed to be conducted. }\end{array}$ \\
\hline The listener's mind & $\begin{array}{l}\text { - Listeners can be shocked or anxious when they learn that someone has a suicide thought. } \\
\text { - It is taught that listeners may have anger, sadness, and desire to deny or avoid that someone has suicidal } \\
\text { thoughts. This psychological reaction may be an unconscious attempt to protect him or her when a person } \\
\text { encounters the suicide thought of other people. }\end{array}$ \\
\hline Unhelpful listening & $\begin{array}{l}\text { - We explain that responding to the emotions of a listener, not the emotions of a person in danger of suicide, } \\
\text { does not help. } \\
\text { - However, it is necessary to provide psychological stability to those who are educated by not directly criticizing } \\
\text { the listener. } \\
\text { - Those who are new to gatekeeper training may have anxiety and fear that things will get worse because of their } \\
\text { inadequate intervention. Therefore, it is necessary to provide psychological stability from time to time. } \\
\text { - It needs to be explained that Gatekeepers should not easily make a promise of secrecy to high-risk people who } \\
\text { commit suicide. } \\
\text { - It should be emphasized that what is more important than secrecy is human life and safety. }\end{array}$ \\
\hline $\begin{array}{l}\text { Question } 2 \text { for listening: } \\
\text { "why did you think about } \\
\text { suicide?" }\end{array}$ & $\begin{array}{l}\text { - It is required to understand their mind in order to help those at risk for suicide. } \\
\text { - Most people think seriously about suicide when they think they are a burden to others or feel isolated. } \\
\text { - You can understand the other person and get more information by sympathetically and directly asking those } \\
\text { who think about suicide. }\end{array}$ \\
\hline $\begin{array}{l}\text { Listening skill: } \\
\text { active listening }\end{array}$ & $\begin{array}{l}\text { - It needs to create an atmosphere in which high-risk people can tell their stories comfortably and honestly. } \\
\text { - Non-judgmental attitude } \\
\text { - You should try to understand the story of people at risk of suicide without judging what is right or wrong. } \\
\text { - Examples of sympathetic listening } \\
\text { - You need to help the person express his feelings the way he wants. } \\
\text { - You should allow the person to talk as much as he wants. } \\
\text { - You have to express your concern to the person. } \\
\text { - You should express verbally and nonverbally that you empathize with the person. }\end{array}$ \\
\hline Gatekeeper's reaction & $\begin{array}{l}\text { - The practice needs to be conducted so that the skills described so far can be applied in practice. } \\
\text { - Examples of empathetic reaction } \\
\text { - "It was so hard that you wanted to die." } \\
\text { - "It must have been tough. I'm sorry that I didn't know. " } \\
\text { - "You can talk to me as much as you want to talk and as much as you can." } \\
\text { - "It must have been a challenging thing for you to tell the story, but thank you for telling me." } \\
\text { - When training is conducted in small classes, trainees can think of and share their empathic expressions with } \\
\text { each other. }\end{array}$ \\
\hline Ambivalence & - People who are thinking of committing suicide may also have a desire to live in their minds. \\
\hline The reason to live & - Paradoxically, the reason to live can be realized by talking about death or the reason we want to die. \\
\hline
\end{tabular}


Table 3. Components \& main contents of the 'risk evaluation and expert referral'

\begin{tabular}{|c|c|}
\hline Item & Core content \\
\hline ideo 3 & $\begin{array}{l}\text { - Situational signals: Difficulties in re-employment, failure to invest using a retiring allowance, physical illness, } \\
\text { wife's cancer diagnosis, disharmony between family members } \\
\text { - Behavioral signals: Sensitivity to minor things, physical symptoms such as stuffiness in the chest, and the act } \\
\text { of throwing away something he has cherished } \\
\text { - Linguistic signals: Direct/indirect expression of death, stories of people who died by suicide, expression of hopelessness }\end{array}$ \\
\hline $\begin{array}{l}\text { Checking suicide } \\
\text { risk }\end{array}$ & $\begin{array}{l}\text { - Asking questions to confirm if he prepares a suicide method and there is any suicide plan } \\
\text { - "Have you ever thought about how to commit suicide?" } \\
\text { - "Have you ever thought when and where to commit suicide?" } \\
\text { - "Have you ever tried suicide before?" } \\
\text {-"When, where, and how have you tried suicide (if any)?" } \\
\text { - If he has a specific plan to commit suicide shortly or has made a fatal suicide attempt, it is an emergent situation. }\end{array}$ \\
\hline Helping safely & $\begin{array}{l}\text { - Securing safety } \\
\text { - Informing quickly family and acquaintances of the information on the suicidal risk } \\
\text { - Not leaving alone him } \\
\text { - Getting rid of lethal means with the prior consent of him } \\
\text { - Refer him to mental health department treatment and hospitalization } \\
\text { - Not recommending alcohol to people at risk of suicide } \\
\text { - } 53.5 \% \text { of suicide attempters who entered the emergency room attempted suicide while drinking. }\end{array}$ \\
\hline $\begin{array}{l}\text { Understanding } \\
\text { depression }\end{array}$ & $\begin{array}{l}\text { - Understanding the connection between suicide and depression } \\
\text { - } 84.5 \% \text { of all suicide deaths had mental illness in their lifetime, and } 62 \% \text { of suicide victims suffered from depression. } \\
\text { - In Korea, the rate of people who receive treatment among those who have depression is just } 15.3 \% \text {. } \\
\text { - Depression is a disease that requires treatment. } \\
\text { - Introduction to the symptoms of depression } \\
\text { - Physical symptoms: insomnia, decreased food intake, fatigue, various physical discomfort } \\
\text { - Mood and cognition: Persistent depression, loss of interest or pleasure, anxiety, difficulty in concentration, excessive } \\
\text { guilt, despair }\end{array}$ \\
\hline $\begin{array}{l}\text { Piece together } \\
\text { a puzzle of hope }\end{array}$ & $\begin{array}{l}\text { - Introduction of people and institutions/services that he can seek help } \\
\text { - Family, friends, and colleagues } \\
\text { - Suicide Prevention Counseling Call (TEL: 1393), 24-hour suicide prevention hotline of the Ministry of Health \& } \\
\text { Welfare (TEL: 1577-0199), Korea Youth Counseling Institute (TEL: 1388), Lifeline Korea (TEL: 1588-9191) } \\
\text { - Korea Suicide Prevention Center, Mental Health Welfare Centers } \\
\text { - Healthcare facilities (Mental Health Department, Emergency Medicine) } \\
\text { - Police Station (TEL: 112), Fire Station (TEL: 119) } \\
\text { - Health and Welfare Counseling Center (TEL: 129) } \\
\text { - Employment Welfare Plus (+) Center, One-stop Credit Support Center (TEL: 1397), Korea Legal Aid Corporation } \\
\text { (TEL: 132), Korea Center on Gambling Problems } \\
\text { - "I" connecting various resources }\end{array}$ \\
\hline Core contacts & $\begin{array}{l}\text { - Suicide prevention counseling call of the Ministry of Health \& Welfare (TEL: 1393) } \\
\text { - 24-hour suicide prevention hotline of the Ministry of Health \& Welfare (TEL: 1577-0199) } \\
\text { - Other information on various welfare services in the country }\end{array}$ \\
\hline Good ending & $\begin{array}{l}\text { - For those who are at risk of suicide, you can reduce the risk of suicide by doing the following: } \\
\text { - Questions about suicide } \\
\text { - Listening sympathetically } \\
\text { - Asking the reasons why they make suicide plans and attempts or why they think about death } \\
\text { - Asking reasons to live and listening carefully } \\
\text { - Recommending to use suicide prevention counseling call (TEL: 1393) } \\
\text { - Prohibition of drinking alcohol, removing lethal means together, promising to live safely }\end{array}$ \\
\hline Role-playing & - Role-playing together with trainees based on the 'Good Ending' script \\
\hline Arrangement & - Arranging the core contents of 'Suicide CARE' \\
\hline
\end{tabular}


was referred to appropriate suicide prevention service without acting suicide attempt. The good finales could be accomplished by making good use of all the skills trainees have learned. In the "Role Play," trainees do a role play based on the "Good Ending" script. In "Arrangement," the core contents of the 'Suicide CARE' are arranged and presented.

\section{DISCUSSION}

The updated 'Suicide CARE' version 2.0. has significant implication for the prevention of suicide in Korea. A lot of recent evidence for the risk and protective factors for suicide have been incorporated into the program. Throughout the brisk discussion among the multidisciplinary team members, the best contents in each category (i.e., "careful observation," "active listening," and "Risk evaluation and Experts referral") were confirmed by consensus. Hence, we believe that the quality of the program has been much improved. Individuals trained to this gatekeeper program is likely to successfully help suicidal people to escape from the suicidal crisis. Together with other preventive strategies, we hope that the 'Suicide CARE' version 2.0 will contribute to decrease of the national suicide rate in Korea.

On the other hand, there are several things to consider for using properly 'Suicide CARE' version 2.0. First, the government should play its role actively. The suicide prevention program using gatekeeper training will be used more widely in the community than in the clinical group. Therefore, it is necessary to provide policy and financial support for the central government and local governments to effectively carry out community-based gatekeeper programs. In other words, the program should not be carried out individually in the private sector, but the government should consistently lead suicide prevention projects. Second, people's perception of suicide and mental health problems should be improved, whereas stigma of those should be eliminated. If there are many misconceptions and prejudices about suicide and mental health problems in local communities, the people at risk of suicide will not seek help. As a result, it will be difficult for those around people at risk of suicide to notice signals of suicide. For the success of the gatekeeper program, there needs to be an active campaign to improve people's perception of suicide and mental health problems and eliminate stigmatization in local communities. Third, a systematic instructor training course should be established and implemented. According to a recently published systematic review, the short-term effect of the gatekeeper program for suicide prevention was excellent, whereas the preventive effects gradually showed a tendency to return to the state before training. ${ }^{22}$ The results provide significant implications in the environment in which gatekeeper training is

implemented for large-scale local community members like Korea. In the end, the quality management of gatekeeper training should be done well, and this can be achieved by standardization and quality control of the instructor training process. Instructor training is mainly conducted for personnel engaged in suicide prevention and the promotion of mental health. Given the amount and number of components of the 'Suicide CARE, eight hours per day would be appropriate for the training. In addition, inactive instructors during a year should receive refresher training. Before the instructor qualification expires, they should be reminded of the qualification period and encouraged to receive refresher training.

One final thing which is required to be performed is to verify the effect of the Korean standard gatekeeper program 'Suicide CARE' version 2.0 on the gatekeeper and suicide high-risk groups through the rigorous study. According to a systematic review of recently conducted gatekeeper training, ${ }^{23}$ studies using randomized controlled trials (RCT) showed that the effect of gatekeeper training on suicide-related knowledge, appraisals, and self-efficacy is unclear. In addition, the quality of many RCTs was evaluated to be low. The objective, composition, and socio-cultural conditions of each gatekeeper program are different. Therefore, we cannot conclude that the gatekeeper program is ineffective by referring only to the results of systematic reviews so far. The controversy about the efficacy of the Gatekeeper program needs to be resolved, and Korea should implement RCT to verify the efficacy of 'Suicide CARE' version 2.0 and confirm the results.

\section{Acknowledgments}

We would like to express our sincere gratitude to Prof. Se-Won Lim for his dedication to the development of "Suicide CARE" versions 1.0 and 1.6. This work was supported by the Standardized Suicide Prevention Program for Gatekeeper Intervention in Korea, version 2.0, a development grant from the Korea Suicide Prevention Center. This work was also supported by the Korea Psychological Autopsy Center. This work was also supported by the Soonchunhyang University Research Fund.

\section{Conflicts of Interest}

The authors have no potential conflicts of interest to disclose.

\section{Author Contributions}

Conceptualization: Hwa-Yong Lee, Hong Jin Jeon, Jong-Woo Paik, Kang Seob Oh. Funding acquisition: Hwa-Yong Lee. Investigation: all authors. Projected administration: all authors. Writing—original draft: Kyoung-Sae $\mathrm{Na}$, Seon Seon-Cheol Park, Hwa-Young Lee, Jong-Woo Paik. Writing-review \& editing: all authors.

\section{ORCID iDs}

Kyoung-Sae Na Seon-Cheol Park Sun-Jung Kwon Minjae Kim Hyoung-Jun Kim Myungjae Baik Jinmi Seol

https://orcid.org/0000-0002-0148-9827 https://orcid.org/0000-0003-3691-4624 https://orcid.org/0000-0001-9938-2237 https://orcid.org/0000-0003-4777-7397 https://orcid.org/0000-0003-0037-4726 https://orcid.org/0000-0001-8989-7405 https://orcid.org/0000-0001-9734-7243 
Eun Ji An Sang Min Lee Eun-Jin Lee Meerae Lim Sung Joon Cho Gwang Hun Kim Nari Kim Hong Jin Jeon Jong-Woo Paik Kang Seob Oh Hwa-Young Lee https://orcid.org/0000-0001-6204-9481 https://orcid.org/0000-0002-7834-8272 https://orcid.org/0000-0002-1579-1007 https://orcid.org/0000-0001-7504-7485 https://orcid.org/0000-0001-6981-0931 https://orcid.org/0000-0002-8407-2663 https://orcid.org/0000-0003-2183-3645 https://orcid.org/0000-0002-6126-542X https://orcid.org/0000-0002-1804-8497 https://orcid.org/0000-0001-9850-1898 https://orcid.org/0000-0002-2749-6232

\section{REFERENCES}

1. Scheidman E. Forward. Oxford, UK: Charles C. Thomas; 1972.

2. Mitchell AM, Kim Y, Prigerson HG, Mortimer-Stephens M. Complicated grief in survivors of suicide. Crisis 2004;25:12-18.

3. Mitchell AM, Sakraida TJ, Kim Y, Bullian L, Chiappetta L. Depression, anxiety and quality of life in suicide survivors: a comparison of close and distant relationships. Arch Psychiatr Nurs 2009;23:2-10.

4. Kinchin I, Doran CM. The economic cost of suicide and non-fatal suicide behavior in the Australian workforce and the potential impact of a workplace suicide prevention strategy. Int J Environ Res Public Health 2017;14.

5. Shepard DS, Gurewich D, Lwin AK, Reed GA Jr, Silverman MM. Suicide and suicidal attempts in the United States: costs and policy implications. Suicide Life Threat Behav 2016;46:352-362.

6. Naghavi M, Global Burden of Disease Self-Harm Collaborators. Global, regional, and national burden of suicide mortality 1990 to 2016: systematic analysis for the Global Burden of Disease Study 2016. BMJ 2019; 364:194.

7. Gjertsen F, Bruzzone S, Griffiths CE. Burden of suicide presented as one of the leading causes of death: uncover facts or misrepresent statistics? J Glob Health 2019;9:010401.

8. Statistics Korea. Causes of death statistics in 2018. Daejeon: Statistics Korea; 2019.

9. OECD. Suicide rates (indicator). Available at: https://data.oecd.org/ healthstat/suicide-rates.htm. Accessed 27 May, 2020.

10. Ministry of Health and Welfare. Act on the Prevention of Suicide and the Creation of Culture of Respect for Life. Sejong: Ministry of Health and Welfare; 2011.

11. Joint Cooperation of Related Departments. National Action Plan for Preventing Suicide. Sejong: Ministry of Health and Welfare; 2018.

12. World Health Organization. Preventing Suicide: A Global Imperative. Geneva: World Health Organization; 2014.

13. Korea Suicide Prevention Center. 2012 Annual Report. Seoul: Korea Suicide Prevention Center; 2013.

14. Korea Suicide Prevention Center. 2014 Annual Report. Seoul: Korea Suicide Prevention Center; 2015.

15. Korea Suicide Prevention Center. 2015 Annual Report. Seoul: Korea Suicide Prevention Center; 2016.

16. Korea Suicide Prevention Center. 2016 Annual Report. Seoul: Korea Suicide Prevention Center; 2017.

17. Ministry of Health and Welfare. "Save a life, It's all our responsibility." Sejong: Ministry of Health and Welfare; 2019.

18. Suicide Prevention Resource Center. Resources and Programs. Available at: http://www.sprc.org/resources-programs?type=All\&populatio $\mathrm{ns}=141$ \&settings $=$ All\&problem $=$ All\&planning $=$ All\&strategies $=89 \&$ st ate $=$ All\&keyword $=$ gatekeeper. Accessed May 28, 2020.

19. Lee SU, Park JI, Lee S, Oh IH, Choi JM, Oh CM. Changing trends in suicide rates in South Korea from 1993 to 2016: a descriptive study. BMJ Open 2018;8:e023144.

20. World Health Organization. Mental Health Atlas 2017 Member State Profile; Republic of Korea. Geneva: World Health Organization; 2018.

21. Park SC, Na KS, Kwon SJ, Kim M, Kim HJ, Baik M, et al. "Suicide CARE" (Standardized Suicide Prevention Program for Gatekeeper Intervention in Korea): an update. Psychiatry Investig 2020;17:911-924.

22. Holmes G, Clacy A, Hermens DF, Lagopoulos J. The long-term efficacy of suicide prevention gatekeeper training: a systematic review. Arch Suicide Res 2019:1-31.

23. Yonemoto N, Kawashima Y, Endo K, Yamada M. Gatekeeper training for suicidal behaviors: a systematic review. J Affect Disord 2019;246: 506-514. 